\title{
Microporous Electrospun Scaffolds for Skin Repair and Regeneration
}

\author{
Paul P. Bonvallet ${ }^{1}$ and Susan L. Bellis ${ }^{1}$ \\ 1. Department of Cell, Developmental, and Integrative Biology, University of Alabama at Birmingham
}

Skin trauma caused by burns, disease or injury can be life-threatening, and skin grafts are often needed to treat large areas of damage. Autograft, allograft and xenograft skin are common graft materials, however all of these have shortcomings, and can elicit scarring, bleeding, infection and/or rejection. A synthetic substitute that recapitulates the characteristics and regenerative potential of native skin would circumvent many of the limitations associated with skin-derived products. The goal of this study was to synthesize scaffolds that mimic the biochemistry, architecture and biomechanical properties of skin. Skin-mimetic scaffolds were produced through electrospinning, a process that generates a 3dimensional, nanofibrous mesh, similar to the structure of native extracellular matrix (ECM). Scaffold nanofibers were composed of a combination of collagen I (col I), the principal component of skin ECM, and the synthetic polymer, polycaprolactone (PCL), which enhances scaffold tensile strength and also allows tuning of scaffold degradation time. Micropores were subsequently introduced into the scaffolds to facilitate cell infiltration and vascular in-growth. The microporous skin-mimetic scaffolds were characterized in vitro for mechanical strength, contractility, and capacity to support the growth of skin cells. Regenerative potential was evaluated by implanting scaffolds into rat full-thickness skin wounds. For some in vivo experiments, scaffolds were seeded with dermal fibroblasts prior to implantation.

Development of skin-mimetic scaffolds with favorable mechanical properties, porosity and capacity to support skin cell survival and differentiation. Pilot studies were first performed to identify the ideal col I to PCL ratio. Scaffolds with varying amounts of col-I and PCL were characterized for tensile properties, contractility and ability to support dermal fibroblast adhesion and proliferation. It was determined that scaffolds composed of $70 \%$ col I and 30\% PCL (70/30 col-PCL) yielded the best balance between mechanical and cell-supportive properties. 70/30 col-PCL scaffolds had a tensile strength of $\sim 2 \mathrm{MPa}$, which is within the lower range of skin tissue, and contractility was $<20 \%$. We next identified an optimal pore diameter, $160 \mu \mathrm{m}$, permissive for cell infiltration. Dermal fibroblasts seeded onto 70/30 col-PCL scaffolds with $160 \mu \mathrm{m}$ micropores migrated into the pores and secreted matrix molecules such as fibrillar collagen and fibronectin, thus remodeling the scaffolds (Fig 1A-C). To assess the capacity of scaffolds to promote cell behaviors critical for wound healing, we developed a co-culture model. Fibroblasts were grown on scaffolds for 10 days to allow matrix deposition, and then keratinocytes were seeded onto the fibroblast-embedded scaffolds (Fig 1 D-E). After growth at an air-liquid interface, the keratinocytes differentiated and formed a stratified layer, similar to native epidermis. CK10 expression, a marker for differentiated keratinocytes, was localized to the apical surface.

Regenerative potential of skin-mimetic scaffolds implanted into rat critical size full-thickness defects. The rate of scaffold degradation is important for proper tissue healing. To evaluate degradation, scaffolds were implanted into rat full-thickness skin wounds, and the amount of scaffold remaining was quantified at varying time points. Scaffolds degraded by 3-4 weeks, which is an optimal time interval for skin wound healing. We next assessed wound healing in rats implanted with 70/30 col-PCL scaffolds with or without $160 \mu \mathrm{m}$ micropores. Relative to sham controls, both the nonporous and microporous scaffolds stimulated superior healing, however the response was significantly better for scaffolds containing the micropores. We subsequently hypothesized that healing might be further enhanced by pre-seeding scaffolds with fibroblasts prior to implantation. Accordingly, microporous 70/30 col-PCL scaffolds with or without pre-seeded fibroblasts were implanted into skin wounds. Analyses of the regenerated tissue following scaffold placement showed that scaffolds with pre-seeded fibroblasts induced a greater amount of ECM with a "basket-weave", rather than scar-like, structure, approximating 
normal skin matrix (Fig 2A-C). As well, the morphology of the epidermal and dermal layers was more similar to native skin, and a larger number of newly-formed blood vessels and hair follicles was observed (Fig 2D). Our collective results suggest that skin-mimetic microporous electrospun scaffolds offer a promising therapeutic alternative to graft materials derived from native skin tissue.

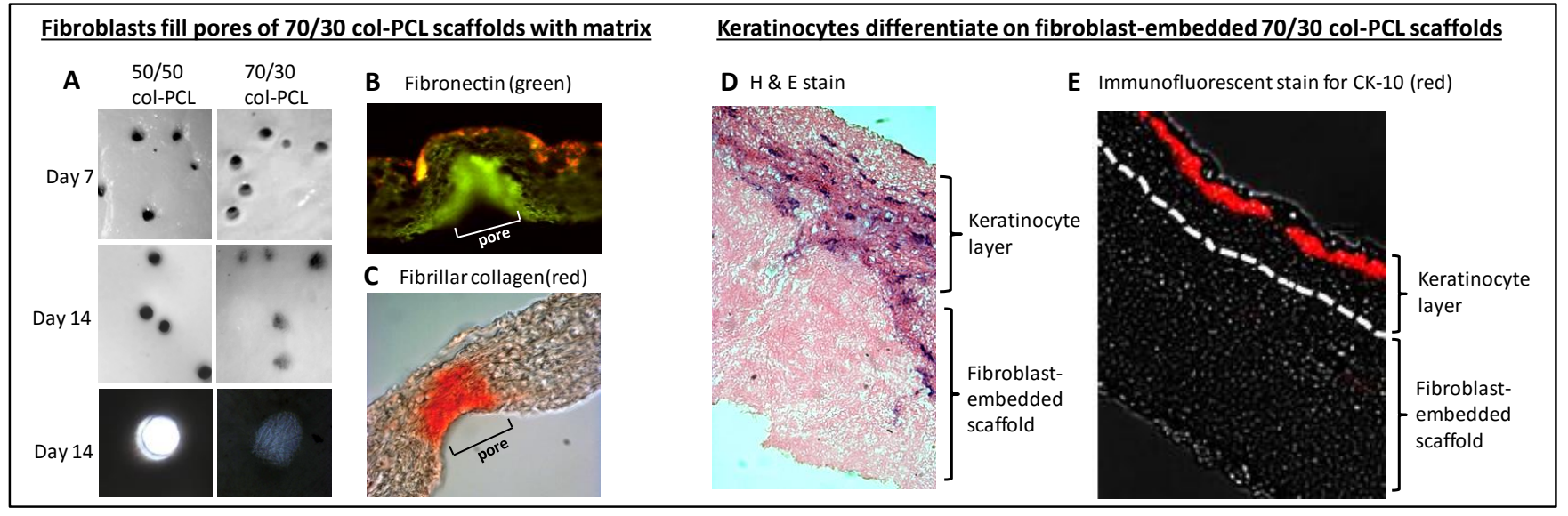

Figure 1. Cell response to scaffolds (A) Dermal fibroblasts were seeded onto microporous scaffolds composed of 50\% col I/50\% PCL (50/50 col-PCL) or 70\% col I/30\% PCL (70/30 col-PCL). After 14 days, fibroblasts deposited fibrous matrix within the pores of the 70/30, but not 50/50, col-PCL scaffolds. (B) Fibroblast-embedded 70/30 col-PCL scaffolds were immunofluorescently stained for fibronectin. (C) Fibroblast-embedded 70/30 col-PCL scaffolds were stained for newly-deposited fibrillar collagen by picrosirius red. (D) Human keratinocytes were seeded onto fibroblastembedded 70/30 col-PCL scaffolds and scaffolds were then grown at an air-liquid interface for 10 days. H\&E staining of the scaffolds showed that keratinocytes formed a stratified layer. (E) Keratinocytes grown for 10 days on fibroblastembedded 70/30 col-PCL scaffolds were immunofluorescently stained for CK-10, a keratinocyte differentiation marker. CK-10 expression was localized to the apical surface of the keratinocyte layer, similar to the known localization of this marker in native epidermis. (Bonvallet et al 2014 Tissue Eng 20: 2434. Copyright permission granted by May Ann Liebert, Inc.)

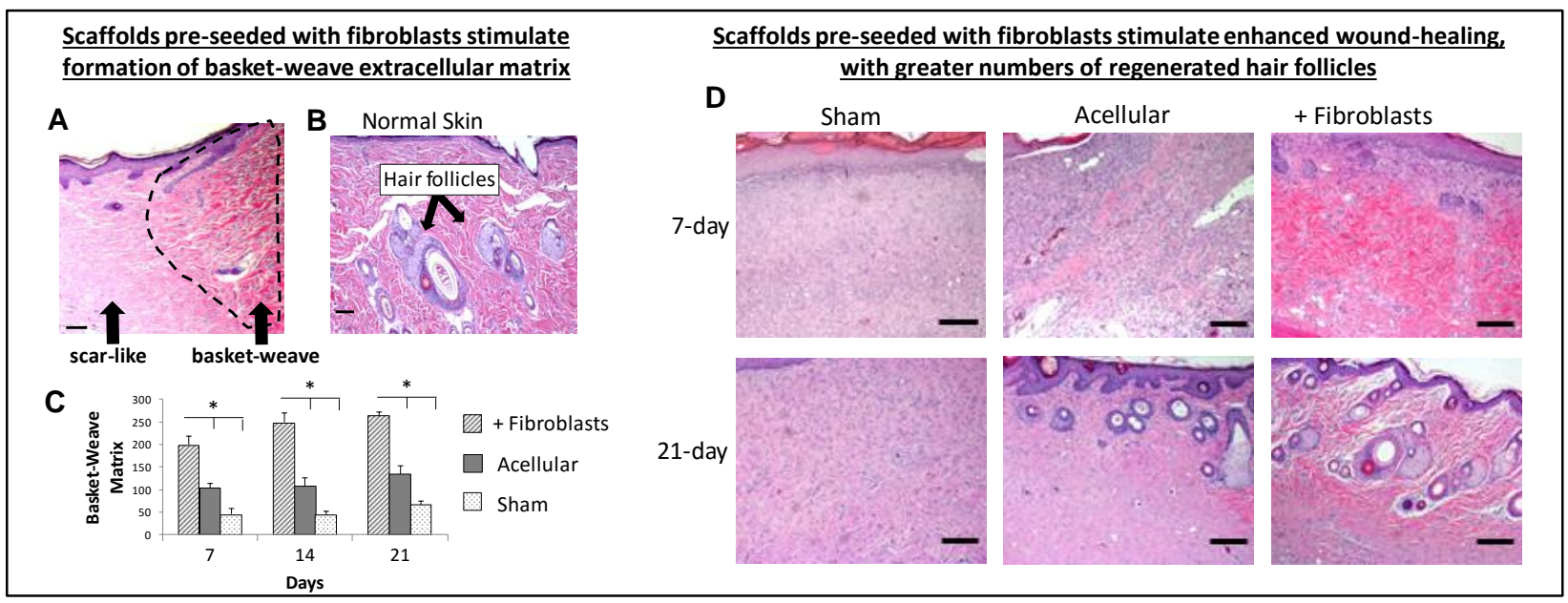

Figure 2. Scaffolds pre-seeded with fibroblasts promote enhanced wound healing. (A) Regenerated matrix following implantation of a 70/30 col-PCL scaffold. Some of the matrix is dense and scar-like, while other areas have a loose, wavy matrix referred to as "basket-weave" matrix (area within dashed lines). Scale bar $=40 \mu \mathrm{m}$. (B) Normal dermis with hair follicles and basket-weave matrix. (C) Quantification of basket-weave matrix shows better healing of wounds implanted with fibroblast-seeded scaffolds (+ fibroblasts) relative to scaffolds lacking fibroblasts (acellular). (D) Images of tissue at 7 or 21 days after implantation with acellular or fibroblast-seeded scaffolds. Shams wounds did not receive implants. Scale bar $=10 \mu \mathrm{m}$. (Bonvallet et al 2015 PLoS One 10: e0122359. Studies published under the Creative Commons Attribution license) 